\title{
Depression as a prodromal symptom of Parkinson Disease
}

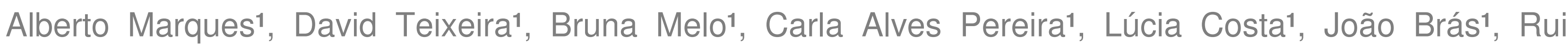 \\ Sousa1, Sofia Ribeiro Pereira ${ }^{1}$
}

1 Department of Psychiatry and Mental Health, Centro Hospitalar Tondela-Viseu, EPE., Viseu, Portugal

OBJECTIVES: To present a clinical case of Parkinson Disease (PD) and its prodromal presentation as a depressive disorder.

BACKGROUND AND AIMS: The cardinal features of Parkinson are resting tremor, rigidity, bradykinesia and impairment of postural reflexes. However, there are several non-motor symptoms that also characterize the disease and not uncommonly precede the appearance of motor signs. The socalled pre-motor symptoms are categorized as autonomic, sensory, sleep-related and psychiatric. The psychiatric manifestations comprise depression, anxiety, apathy, dementia, hallucinations and impulse control disorders. Depression is the most frequent among these, affecting $33-50 \%$ of PD patients. Presenting with non-motor symptoms is associated with a delayed diagnosis.

\begin{tabular}{|c|c|}
\hline \multicolumn{2}{|c|}{ Non-Motor Sleep-Related Symptoms } \\
\hline Sleep Fragmentation & Day-time Sleepiness \\
\hline REM Behaviour Disorder & Altered Sleep-Wake Cycle \\
\hline \multicolumn{2}{|c|}{ Drug-Induced Sleep-Attacks (Dopaminergic Agents) } \\
\hline
\end{tabular}

\begin{tabular}{|c|c|}
\hline \multicolumn{2}{|c|}{ Non-Motor Psychiatric Symptoms } \\
\hline Depression & Anxiety \\
\hline Apathy & Dementia \\
\hline Psychosis: Hallucinations, Paranoid Delusions (often infidelity) \\
\hline \multicolumn{2}{|c|}{ Impulse Control Disorders: Pathologic Gambling, } \\
Hypersexuality, Compulsive Buying, Binge-Eating, Punding \\
\hline
\end{tabular}

MATERIALS AND METHODS: Patient's clinical files consultation and scientific bibliography search using the keywords Parkinson Disease, Non-motor Symptoms, Depression in Parkinson Disease.

RESULTS: A 74 year-old woman, with no previous psychiatric history, was diagnosed with major depressive disorder. The symptoms began after a highly disruptive life-event: a house fire with great material losses. Although the mood disorder successfully responded to anti-depressant medication, the patient kept complaining about physical distress. One year into the follow-up she showed all cardinal parkinsonic features.

\begin{tabular}{|c|c|}
\hline \multicolumn{2}{|c|}{ Non-Motor Autonomic Symptoms } \\
\hline Rhinorrhea & Sexual Disfunction \\
\hline Dyspnea & Sialorrhea \\
\hline Seborrhea & Sweating \\
\hline \multicolumn{2}{|c|}{ Hypotension: postural, post-prandial } \\
\hline \multicolumn{2}{|c|}{ Bladder: nocturia, frequency, urgency } \\
\hline Gastrointestinal: dysphagia, constipation, abdominal bloating \\
\hline
\end{tabular}

\begin{tabular}{|c|c|}
\hline \multicolumn{2}{|c|}{ Non-Motor Sensory Symptoms } \\
\hline Pain & Burning \\
\hline Numbness & Paresthesias \\
\hline Akathisia & Hyposmia \\
\hline \multicolumn{2}{|c|}{ Restless Legs Syndrome } \\
\hline
\end{tabular}

> Non-Motor Syptoms are usually a greater source of disconfort than Motor ones and the most troublesome for PD patients.

$>$ When it comes to balancing drugs in PD, keep in mind that an intact mental function is more important than an intact motor function.

CONCLUSIONS: Late onset depression, even if in the context of comprehensible disruptive life events, deserves close follow-up. Depression can present as a pre-motor manifestation of PD, in some cases several years prior to diagnosis. Therefore, a first depressive episode late in life should raise suspicion for an underlying neurodegenerative disorder. The longitudinal evaluation of the patients' semiology is key to a correct diagnosis and early neurology referral. 